\title{
Compact Encodings of Planar Orthogonal Drawings ${ }^{\star}$
}

\author{
Amrita Chanda and Ashim Garg \\ Department of Computer Science and Engineering \\ University at Buffalo \\ Buffalo, NY 14260 \\ \{achanda, agarg\}@cse. buffalo.edu
}

\begin{abstract}
We present time-efficient algorithms for encoding (and decoding) planar orthogonal drawings of degree- 4 and degree- 3 biconnected and triconnected planar graphs using small number of bits. We also present time-efficient algorithms for encoding (and decoding) turn-monotone planar orthogonal drawings.
\end{abstract}

\section{Introduction}

It is important to compress the representation of planar orthogonal drawings to reduce their storage requirements and transmission times over a network, like Internet. The encoding problem is also interesting from a theoretical viewpoint.

A degree- $d$ graph is one, where each vertex has at most $d$ edges incident on it. A planar drawing is one with no edge-crossings. An orthogonal drawing is one, where each edge is drawn as an alternating sequence of horizontal and vertical line-segments. A bend (turn) is defined as the meeting point of two consecutive line-segments of an edge in a drawing.

In this paper, we investigate the problem of encoding planar orthogonal drawings of degree- 4 and degree- 3 biconnected and triconnected planar graphs using small number of bits, and present several results.

Let $d$ be a planar orthogonal drawing, with $b$ turns (bends), of a planar graph $G$ with $n$ vertices, $m$ edges, and $f$ internal faces. Suppose each line-segment of $d$ has length at most $W$. Through out the paper, to avoid triviality, we will assume that $n \geq 3$. A simple drawing-description format that stores the underlying graph using adjacency-list representation, and stores the coordinates of the vertices and edge-bends would require $\Omega\left(n+m \log _{2} n+n \log _{2}(b+n) W+b \log _{2}(b+n) W\right)$ bits in the worst case. More complex formats may require even more bits. We are not aware of any work focusing explicitly on encoding planar orthogonal drawings.

In Sections 2 3, and 4, respectively, we give our result, previous work, and some definitions. In Sections 50 and 6 respectively, we show how to encode (and decode) degree-3 and degree-4 plane graphs, orthogonal representations, and lengths of the line-segments of a planar orthogonal drawing. In Section 8 , we give our overall algorithm for encoding (and decoding) a planar orthogonal drawing.

\footnotetext{
* Research supported by NSF CAREER Award IIS-9985136 and NSF CISE Research
} Infrastructure Award No. 0101244. 


\section{Our Result}

Our results can be summarized as follows (with $G, n, m, f, d, W$, and $b$ as defined above): Let $p=(2+b+m-f) \log _{2}(W+1)$. Let $q=n+b$.

- If $G$ is a degree- 4 biconnected graph, then we can encode (and decode) $d$ using at most $4.74 m+2.42 n+1.58 b+p+O(\log q)$ bits in $O\left((n+b+p)^{2}\right)$ time, and using at most $5.01 m+2.33 n+1.67 b+p+O(\log q)$ bits in $O(n+b+p)$ time.

- If $G$ is a degree- 4 triconnected graph, then we can encode (and decode) $d$ using at most $3.58 m+2.59 n+1.58 b+p+O(\log q)$ bits in $O\left((n+b+p)^{2}\right)$ time, and using at most $3.67 m+2.67 n+1.67 b+p+O(\log q)$ bits in $O(n+b+p)$ time.

- If $G$ is a degree-3 biconnected graph, then we can encode (and decode) $d$ using at most $4.74 m+1.23 n+1.58 b+p+O(\log q)$ bits in $O\left((n+b+p)^{2}\right)$ time, and using at most $5.01 m+1.33 n+1.67 b+p+O(\log q)$ bits in $O(n+b+p)$ time.

- If $G$ is a degree-3 triconnected graph, then we can encode (and decode) $d$ using at most $3.58 m+n+1.58 b+p+O(\log q)$ bits in $O\left((n+b+p)^{2}\right)$ time, and using at most $3.67 m+n+1.67 b+p+O(\log q)$ bits in $O(n+b+p)$ time.

Several drawing algorithms 78 , that try to minimize the number of bends, produce turn-monotone planar orthogonal drawings, i.e., where for each edge $e=(u, v)$, if we travel from $u$ to $v$ along $e$, then we will either make left turns or right turns, but not both (see Figure 1(a)). Turn-monotone planar orthogonal drawings are very common in practice. We show that a turn-monotone planar orthogonal drawing $d$ can be encoded even more succinctly:

- If $G$ is a degree- 4 biconnected graph, then we can encode (and decode) $d$ using at most $3.16 m+4 n+1.58 b+p+O(\log q)$ bits in $O\left((n+b+p)^{2}\right)$ time, and using at most $3.34 m+4 n+1.67 b+p+O(\log q)$ bits in $O(n+b+p)$ time.

- If $G$ is a degree- 4 triconnected graph, then we can encode (and decode) $d$ using at most $2 m+4.17 n+1.58 b+p+O(\log q)$ bits in $O\left((n+b+p)^{2}\right)$ time, and using at most $2 m+4.34 n+1.67 b+p+O(\log q)$ bits in $O(n+b+p)$ time.

- If $G$ is a degree-3 biconnected graph, then we can encode (and decode) $d$ using at most $3.16 m+2.81 n+1.58 b+p+O(\log q)$ bits in $O\left((n+b+p)^{2}\right)$ time, and using at most $3.34 m+3 n+1.67 b+p+O(\log q)$ bits in $O(n+b+p)$ time.

- If $G$ is a degree-3 triconnected graph, then we can encode (and decode) $d$ using at most $2 m+2.58 n+1.58 b+p+O(\log q)$ bits in $O\left((n+b+p)^{2}\right)$ time, and using at most $2 m+2.67 n+1.67 b+p+O(\log q)$ bits in $O(n+b+p)$ time.

As a by-product, our technique also encodes orthogonal representations, which are important intermediate constructs used by several drawing algorithms [7].

\section{Related Work}

As mentioned above, we are not aware of any previous work focusing explicitly on encoding planar orthogonal drawings. However, a lot of work has been done on encoding planar graphs. Let $G$ be a planar graph with $n$ vertices and $m$ edges. If $G$ is biconnected (triconnected, triangulated, respectively), then it can be 
encoded using at most $2 n+1.58 m$ bits [1] $(1.58(n+m)$ bits [1], $1.33 m$ bits [1]). If $G$ is a triangulated graph, then any encoding of $G$ requires at least $1.08 \mathrm{~m}$ bits [9]. [5] presents a technique for encoding $G$ in asymptotically the minimum number of bits in $O(n \log n)$ time. For more results on graph encoding, see [1].

Our encoding technique is based on the graph encoding technique of [1], and on the concept of canonical orderings of planar graphs $[2,6,1]$.

\section{Preliminaries}

We use standard definitions of graph-theoretic terms. A plane graph $G$ is a planar graph equipped with an embedding. Two vertices of $G$ are neighbors if they are connected by an edge. Let $u_{1}, u_{2}, \ldots, u_{k}$ be some vertices of $G$. The plane graph induced by $u_{1}, u_{2}, \ldots, u_{k}$ is the maximal subgraph of $G$ that consists of these vertices and their incident edges. Suppose $G$ has $n$ vertices. An ordering $v_{1}, v_{2}, \ldots, v_{n}$ of the vertices of $G$ is an assignment of unique integers in the range $[1, n]$ to the vertices, such that the $i^{t h}$ vertex $v_{i}$ in the order is assigned number $i$.

Let $G$ be a degree-4 plane graph. Two planar orthogonal drawings $\Gamma_{1}$ and $\Gamma_{2}$ of $G$ are shape equivalent if: (1) for each vertex $v$, consecutive edges incident to $v$ form the same angle at $v$ in $\Gamma_{1}$ and $\Gamma_{2}$, and (2) for each edge $(u, v)$, the sequence of left and right turns encountered while walking from $u$ to $v$ along the polygonal chain representing $(u, v)$ is the same in $\Gamma_{1}$ and $\Gamma_{2}$. An orthogonal representation $\Gamma$ of $G$ defines an equivalence class of shape equivalent planar orthogonal drawings of $G . \Gamma$ is a turn monotone representation if each edge is represented as a polygonal chain consisting of only left or right turns, but not both (see Figure 1(a)).

An important concept used by our encoding technique is that of a canonical ordering of a plane graph (see Figure 1(b)). This concept has been defined and used in $[2,6,1]$. Let $G=(V, E)$ be a simple biconnected plane graph with $n$ vertices,

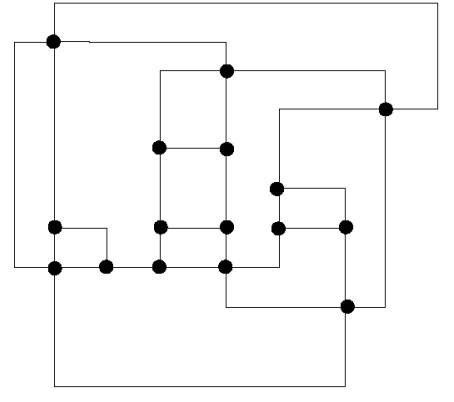

(a)

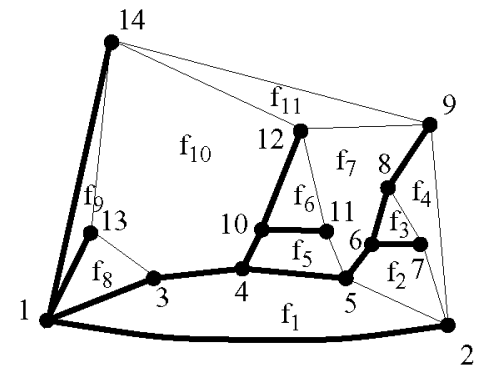

(b)

Fig. 1. (a) A turn-monotone planar orthogonal drawing. (b) corresponding plane graph $G$, a rightmost canonical ordering $c$ of $G$ and the canonical spanning tree $T_{c}$ corresponding to $c$. Here, each vertex is labeled by its number in $c$, the edges of $T_{c}$ are shown as dark lines. Each face is also labeled by its number in corresponding ordering of faces. 
and $m$ edges. Let $v_{1}, v_{2}, \ldots, v_{n}$ be an ordering of the vertices of $G$. Let $G_{i}$ be the plane graph induced by vertices $v_{1}, v_{2}, \ldots, v_{i}$. Let $H_{i}$ be the external face of $G_{i}$.

Definition 1 ([1]). Let $v_{1}, v_{2}, \ldots, v_{n}$ be an ordering of the vertices of a biconnected plane graph $G=(V, E)$, where $v_{1}$ and $v_{2}$ are two arbitrary vertices on the external face of $G$ with $\left(v_{1}, v_{2}\right) \in E$. The ordering is canonical if there exist ordered partitions $I_{1}, I_{2}, \ldots, I_{K}$ of the interval $[3, n]$ such that the following properties hold for every $1 \leq j \leq K$ : Suppose $I_{j}=[k, k+q]$. Let $C_{j}$ be the path $\left(v_{k}, v_{k+1}, \ldots, v_{k+q}\right)$ (Note, $C_{j}$ is a single vertex if $\left.q=0\right)$. Suppose we say that a vertex $u$ of $G_{k-1}$ is a neighbor of $C_{j}$ if a vertex of $C_{j}$ is a neighbor of $u$. Then:

- The graph $G_{k+q}$ is biconnected. Its external face $H_{k+q}$ contains the edge $\left(v_{1}, v_{2}\right)$, and the path $C_{j} . C_{j}$ has no chord in $G$, i.e., $G$ does not contain any edge $\left(v_{s}, v_{t}\right)$ with $|s-t|>1$ and $k \leq s, t \leq k+q$.

- $C_{j}$ has at least two neighbors in $G_{k-1}$, all of which are vertices of $H_{k-1}$. The leftmost neighbor is a neighbor of $v_{k}$ and the rightmost neighbor is a neighbor of $v_{k+q}$. Moreover, if $q>0$, then $v_{l}$ and $v_{r}$ are the only neighbors of $C_{j}$ in $G_{k-1}$. Here, the leftmost and rightmost neighbors of $C_{j}$ in $G_{k-1}$ are defined as follows: Vertices $v_{1}$ and $v_{2}$ divide $H_{k-1}$ into two paths: a path consisting only of edge $\left(v_{1}, v_{2}\right)$, and another path $v_{1}\left(=u_{1}\right) u_{2} \ldots u_{s}\left(=v_{2}\right)$ that connect $v_{1}$ and $v_{2}$, and that does not contain the edge $\left(v_{1}, v_{2}\right)$. We say that a vertex $u_{i}$ is left (right) of another vertex $u_{z}$, if $i<z(i>z)$. The leftmost (rightmost) neighbor of $C_{j}$ in $G_{k-1}$ is the vertex $u_{b}$ such that $u_{b}$ is a neighbor of $C_{j}$, and there is no other vertex $u_{t}$ such that $u_{t}$ is a neighbor of $C_{j}$ and $u_{t}$ is left (right) of $u_{b}$.

A canonical ordering for a triconnected plane graph is defined the same as for a biconnected plane graph, except that it has the following additional property:

Property 1 ([1]). Every vertex $v_{k}$, where $1 \leq k \leq n-1$ has at least one neighbor $v_{p}$ with $k<p$.

A rightmost canonical (rmc) ordering for a biconnected plane graph $G$ is defined as follows (see Figure 1(b)):

Definition 2 ([1]). Let $v_{1}, v_{2}, \ldots, v_{n}$ be a canonical ordering for $G$, where $I_{1}, I_{2}, \ldots, I_{K}$ are its corresponding interval partitions. We say that $v_{1}, v_{2}, \ldots, v_{n}$ is a rightmost canonical ( $r m c$ ) ordering for $G$ if the following property holds for every interval $I_{j}$, where $1 \leq j \leq K$ :

Suppose $I_{j}=[k, k+q]$. Let $v_{1}, v_{2}, \ldots, v_{k-1}, u_{k}, u_{k+1}, \ldots, u_{n}$ be any canonical ordering for $G$ whose first $j-1$ interval partitions are exactly $I_{1}, I_{2}, \ldots, I_{j-1}$ (Clearly, the $G_{k-1}$ and $H_{k-1}$ with respect to both canonical orderings are the same). Let $v_{l}$ be the leftmost neighbor of $v_{k}$ on $H_{k-1}$. Let $u_{l}$ be the leftmost neighbor of $u_{k}$ on $H_{k-1}$. Then, $v_{l}$ is to the right of $u_{l}$ on $H_{k-1}$.

Theorem 1. [1] Every biconnected plane graph $G$ with $n$ vertices admits a rightmost canonical (rmc) ordering, which can be constructed in $O(n)$ time. 
Any canonical ordering $c=v_{1}, v_{2}, \ldots, v_{n}$ of a biconnected plane graph $G$ and its corresponding interval partitions $I_{1}, I_{2}, \ldots, I_{K}$ defines a canonical spanning tree $T_{c}$ that consists of the edge $\left(v_{1}, v_{2}\right)$ plus the union of the paths $v_{l} v_{k} v_{k+1} \ldots v_{k+q}$ over all intervals $I_{j}=\left[v_{k}, v_{k+q}\right]$, where $1 \leq j \leq K$ and $v_{l}$ is the leftmost neighbor of $v_{k}$ on $H_{k-1}$ (see Figure $1(\mathrm{~b})$ ). Suppose we root $T_{c}$ at $v_{1}$. A tree edge of a vertex $v$ is one that also belongs to $T_{c}$, and a non-tree edge is one that does not. The incoming tree edge of $v$ is the edge that connects $v$ to its parent in $T_{c}$, and an outgoing tree edge is one that connects $v$ to a child in $T_{c}$. Suppose vertex $v$ belongs to interval $I_{j}=\left[v_{k}, v_{k+q}\right]$. The incoming non-tree edges of $v$ are those that connect $v$ to neighbors in $G_{k-1}$, and the outgoing non-tree edges are the remaining non-tree edges. Note that by the definition of canonical ordering, each outgoing non-tree edge of $v$ will be of the form $\left(v, v_{s}\right)$, where $s>k+q$. Also, note that for each incoming non-tree edge $(u, v)$ of $v, u$ will be on $H_{k-1}$. For example, in Figure 1(b), Vertex 12 has incoming tree edge $(10,12)$, no outgoing tree edge, incoming non-tree edges $(11,12)$ and $(9,12)$, and outgoing non-tree edge $(12,14)$.

Properties 2 and 3 follow easily from the definition of canonical ordering:

Property 2. Let $v \neq v_{1}$ be a vertex of $G$. Then, $v$ has exactly one incoming tree edge, and at least one outgoing tree-edge or incoming non-tree edge. Vertex $v_{1}$ has only outgoing tree edges.

Property 3. If $G$ is a triconnected graph, then for every vertex $v \neq v_{1}, v_{n}$ of $G, v$ has Property 2. Also, it has either at least one outgoing tree-edge, or at least one outgoing non-tree edge. Vertex $v_{1}$ has only outgoing tree edges. Vertex $v_{n}$ has exactly one incoming tree edge and all its other edges are incoming non-tree edges.

Theorem 2. Let $G$ be a biconnected plane graph with $n$ vertices. Suppose, we are given a rightmost canonical ordering $c=v_{1}, v_{2}, \ldots, v_{n}$ of the vertices of $G$, along with the number of outgoing tree edges, incoming non-tree edges, and outgoing non-tree edges of each vertex as defined by $c$. Then, we can determine in $O(n)$ time, all the edges in $G$, as well as its embedding. In other words, given this information, we can determine the entire structure of graph $G$.

Sketch of Proof. Starting from the empty graph $G_{0}$, we reconstruct $G$ iteratively by constructing a sequence of plane graphs $G_{0}, G_{1}, G_{2}, \ldots, G_{p}=G$, where in each iterative step $i$, we obtain $G_{i}$ from $G_{i-1}$ by inserting some vertices and edges into it. This is done as follows: For each vertex $v_{k}$, where $1 \leq k \leq n$, maintain two counters, TCount $\left(v_{k}\right)$ and $N \operatorname{count}\left(v_{k}\right)$, that initially store the number of outgoing tree edges and outgoing non-tree edges, respectively, of $v_{k}$.

In the first iterative step, insert into $G_{0}$, vertices $v_{1}, v_{2}, \ldots, v_{r}$, where $v_{r}$ is the lowest numbered vertex in $c$ with an incoming non-tree edge. Also, insert the edges $\left(v_{1}, v_{2}\right),\left(v_{1}, v_{3}\right),\left(v_{3}, v_{4}\right), \ldots,\left(v_{r-1}, v_{r}\right),\left(v_{r}, v_{2}\right)$. Note that these edges will form a single simple cycle. Reduce $N \operatorname{count}\left(v_{2}\right)$ by 1 . For each $j$, where $1 \leq j \leq r-1$ and $j \neq 2$, reduce $T \operatorname{Count}\left(v_{j}\right)$ by 1 .

In a general iterative step $i$, we obtain $G_{i}$ from $G_{i-1}$ as follows: Suppose $G_{i-1}$ consists of vertices $v_{1}, v_{2}, \ldots, v_{k-1}$. Let $v_{k}, v_{k+1}, \ldots, v_{k+q}$ be the sequence 
of consecutive vertices in $c$ such that vertices $v_{k}, v_{k+1}, \ldots, v_{k+q-1}$ do not have any incoming non-tree edge, but $v_{k+q}$ does. Let $H_{k-1}$ be the external face of $G_{k-1}$. Let $u$ be the rightmost vertex on $H_{k-1}$ with $T$ Count $(u)>0$. We have 2 cases:

$-q=0$ : Suppose $v_{k}$ has $t$ incoming non-tree edges. Let $u_{1}, u_{2}, \ldots, u_{t}$ be the first $t$ vertices to the right of $u$ on $H_{k-1}$, such that, for each $u_{i}, N \operatorname{Count}\left(u_{i}\right)>$ 0 . Insert vertex $v_{k}$ and the edges $\left(u, v_{k}\right),\left(v_{k}, u_{1}\right),\left(v_{k}, u_{2}\right), \ldots,\left(v_{k}, u_{t}\right)$ into $G_{k-1}$ to obtain $G_{k}$. For each $i$, where $1 \leq i \leq t$, reduce $N \operatorname{Count}\left(u_{i}\right)$ by 1 (Note that except for $u_{t}, N \operatorname{Count}\left(u_{i}\right)$ for each $u_{i}$ will become 0 now). Reduce TCount $(u)$ by 1 .

$-q>0$ : In this case, $v_{k+q}$ will have only one neighbor $u^{\prime}$ on $H_{k-1}$, where $u^{\prime}$ is the first vertex right of $u$ with $N$ Count $\left(u^{\prime}\right)>0$. Insert vertices $v_{k}, v_{k+1}, \ldots$, $v_{k+q}$ and edges $\left(u, v_{k}\right),\left(v_{k}, v_{k+1}\right),\left(v_{k+1}, v_{k+2}\right), \ldots,\left(v_{k+q-1}, v_{k+q}\right),\left(v_{k+q}, u^{\prime}\right)$, into $G_{k-1}$ to obtain $G_{k}$. Reduce $N$ Count $\left(u^{\prime}\right)$ by 1 . Reduce TCount $(u)$ by 1 . Also, for each $i$, where $k \leq i \leq k+q-1$, reduce TCount $\left(v_{i}\right)$ by 1 .

\section{Encoding Degree-3 And Degree-4 Plane Graphs}

The algorithms of [1] will encode a biconnected (triconnected) degree-4 plane graph using $5.17 n$ bits ( $4.74 n$ bits), and a degree-3 biconnected (triconnected) plane graph using $4.37 n(3.95 n)$ bits. But, these algorithms do not consider the degrees of vertices while encoding a graph. (The algorithm of 5 will construct an asymptotically bit-minimum encoding of these graphs, but it is practical only for very large graphs.) Here, we show that we can get a better encoding for degree-3 and degree- 4 plane graphs by considering the degrees of their vertices.

The basic idea is simple. Suppose we construct a rightmost canonical ordering $c=v_{1}, v_{2}, \ldots, v_{n}$ of the vertices of a biconnected plane graph $G$. Then, to encode $G$, from Theorem 2, it is sufficient to encode, for each vertex, how many outgoing tree edges, incoming non-tree edges, and outgoing non-tree edges the vertex has.

Suppose $G$ is a degree-3 graph. From Property 2] a vertex $v \neq v_{1}$ can only be of one of the 7 types, $A$ to $G$, based on its degree, and the number and types of its edges. $v$ is of one of types $A$ to $F$, if it has degree 3 , and of type $\mathrm{F}$ or $\mathrm{G}$ if it has degree 2. $v$ is of (a) Type A: if it has two outgoing tree edges, (b) Type B: if it has one outgoing tree edge and one incoming non-tree edge, (c) Type $C$ : if it has one outgoing tree edge and one outgoing non-tree edge, (d) Type D: if it has one incoming and one outgoing non-tree edge, (e) Type E: if it has two incoming non-tree edges, (f) Type $F$ : if it has one incoming non-tree edge, and (g) Type $G$ : if it has one outgoing tree edge. Note that vertex $v_{1}$ will have either two or three outgoing tree edges. Thus, we encode $G$ by a string $S=s_{1} s_{2} \ldots s_{n}$, where

$-s_{1}$ represents the number of outgoing tree edges of $v_{1}$, and is equal to 0 if $v_{1}$ has two outgoing edges, and is equal to 1 if $v_{1}$ has three outgoing edges.

- Each symbol $s_{i}, 2 \leq i \leq n$, represents the type of vertex $v_{i}$, and is equal to $A, B, C, D, E, F$, or $G$.

Since each $s_{i}$, where $2 \leq i \leq n$, can have 7 possible values, we can encode the substring $S^{\prime}=s_{2} \ldots s_{n}$ using $(n-1) \log _{2} 7 \approx 2.81(n-1)$ bits by converting the 
corresponding Base-7 number into binary representation in $O\left(n^{2}\right)$ time. Using Huffman encoding, we can encode $S^{\prime}$ using at most $3(n-1)$ bits in $O(n)$ time. This, combined with Theorems 1 and 2 , gives us the following lemma:

Lemma 1. Given a degree-3 biconnected plane graph $G$ with $n$ vertices, we can encode it using less than $2.81 n$ bits in $O\left(n^{2}\right)$ time and decode the encoding to reconstruct $G$ in $O\left(n^{2}\right)$ time. We can also encode $G$ using at most $3 n-2$ bits and decode the encoding to reconstruct $G$ in $O(n)$ time.

If $G$ is a degree-3 triconnected graph, then, using Property 3 , we can show that each vertex $v \neq v_{1}, v_{n}$ can only be of 4 types. This gives a shorter encoding for $G$ :

Lemma 2. Given a degree-3 triconnected plane graph $G$ with $n$ vertices, we can encode it using at most $2 n-2$ bits in $O(n)$ time (using Huffman Encoding). This encoding can be decoded in $O(n)$ time to reconstruct $G$.

If $G$ is a degree- 4 biconnected (triconnected) graph, then each vertex $v$, where $v \neq v_{1}\left(v \neq v_{1}, v_{n}\right)$, can be of 16 (12) types. This gives Lemma 3 (Lemma 4):

Lemma 3. Given a degree-4 biconnected plane graph $G$ with $n$ vertices, we can encode it using at most $4 n-2$ bits in $O(n)$ time (using Huffman Encoding) and decode the encoding to reconstruct $G$ in $O(n)$ time.

Lemma 4. Given a degree-4 triconnected plane graph $G$ with $n$ vertices, we can encode it using $2+(n-2) \log _{2} 12+1<3.59 n$ bits in $O\left(n^{2}\right)$ time and decode the encoding to reconstruct $G$ in $O\left(n^{2}\right)$ time. We can also encode $G$ using at most $2+3.67(n-1)+1<3.67 n$ bits (using Huffman encoding) and decode the encoding to reconstruct $G$ in $O(n)$ time.

\section{Encoding an Orthogonal Representation}

We will use the following properties of an orthogonal representation:

Property 4. The sum of angles around any vertex is equal to $360^{\circ}$.

Property 5. The sum of interior angles of the polygon $p$ representing any internal face is equal to $(k-2) 180^{\circ}$, where $k$ is the total number of line-segments in $p$.

We can encode an orthogonal representation $\Gamma$ of a biconnected plane graph $G$ by:

- encoding structure: encoding the structure of graph $G$,

- encoding angles: for each vertex, encoding the angles between consecutive edges incident on it, and

- encoding turns: for each edge $e=(u, v)$, encoding the sequence of left and right turns encountered while walking from $u$ to $v$ along $e$. 
To encode angles, suppose $G$ has $n$ vertices and $m$ edges. Each angle of $\Gamma$ can be either $90^{\circ}, 180^{\circ}$, or $270^{\circ}$. Suppose we have already constructed a rightmost canonical ordering $c=v_{1}, v_{2}, \ldots, v_{n}$ of the vertices of $G$. Let $v_{i}$ be a vertex of $G$. Let $e_{1} e_{2} \ldots e_{k}$, where $k \leq 4$ be the counterclockwise order of edges incident on $v_{i}$, where, if $v_{i} \neq v_{1}$, then $e_{1}$ is the incoming tree edge of $v$, and if $v_{i}=v_{1}$, then $e_{1}$ is the edge $\left(v_{1}, v_{2}\right)$. Let $s_{i}^{*}$ be the string $a_{1} a_{2} \ldots a_{k}$, where $a_{j}$ represents the counterclockwise angle between edges $e_{j}$ and $e_{j+1}$ at vertex $v_{i} . a_{j}$ is equal to $A$, $B$, or $C$, respectively, if the magnitude of the corresponding angle is equal to $90^{\circ}$, $180^{\circ}$, or $270^{\circ}$, respectively. Then, we can construct a string $S^{*}=s_{1}^{*} s_{2}^{*} \ldots s_{n}^{*}$, that encodes all the angles of $G$. Total number of symbols in $S^{*}$ is equal to number of angles in $\Gamma$, which is equal to $2 m$.

Using Property 4, we can encode $S^{*}$ using even fewer bits. Property 4 implies that, for each vertex $v_{i}$, it is sufficient to encode angles $a_{1}, a_{2}, \ldots, a_{k-1}$ since the value of angle $a_{k}$ can be obtained from them. Thus, for $v_{i}$, it is sufficient to construct the string $s_{i}^{*}=a_{1} a_{2} \ldots a_{k-1}$. So, the overall number of symbols in string $S^{*}$ can be reduced to $2 m-n$. We therefore have the following lemma:

Lemma 5. Given an orthogonal representation of a degree-4 biconnected graph $G$ with $n$ vertices, we can encode its angles using $(2 m-n) \log _{2} 3 \approx 1.58(2 m-n) \leq$ $4.74 n$ bits in $O\left(n^{2}\right)$ time and at most $1.67(2 m-n) \leq 5.01 n$ bits in $O(n)$ time (using Huffman Encoding). Moreover, during decoding, if we already knew the degree of each vertex, then we can decode these encodings to obtain the angles in $O\left(n^{2}\right)$ and $O(n)$ time, respectively.

If $G$ is a triconnected graph, then each vertex has at least 3 angles around it, and so each angle can be either $90^{\circ}$, or $180^{\circ}$. Therefore:

Lemma 6. Given an orthogonal representation of a degree-4 triconnected graph $G$ with $n$ vertices, we can encode its angles using $2 m-n$ bits in $O(n)$ time. Moreover, during decoding, if we already knew the degree of each vertex, then we can decode the encoding to obtain the angles in $O(n)$ time.

Now consider the problem of encoding the turns of $\Gamma$. Given a rightmost canonical ordering $c=v_{1}, v_{2}, \ldots, v_{n}$ of $G$, and the associated canonical tree $T_{c}$, we first construct an ordering $o=e_{1}, e_{2}, \ldots, e_{m}$ of the edges of $G$ by putting the incoming tree and non-tree edges of the vertices $v_{1}, v_{2}, \ldots, v_{n}$ into $o$, such that the edges of $v_{i}$ precede those of $v_{j}$ if $i<j$, and for each vertex $v_{i}$, we first put its incoming tree edge, and then its incoming non-tree edges in the same order as their counter-clockwise order around $v_{i}$. Next, for each edge $e_{i}=\left(v_{j}, v_{k}\right)$ in $o$, where $j<k$, we construct a (possibly empty) string $s_{i}^{+}$consisting of symbols $L$ and $R$, where a symbol $L(R)$ denotes a left (right) turn encountered while walking from $v_{j}$ to $v_{k}$ along $e_{i}$. These symbols are placed in $s_{i}^{+}$in the order the corresponding turns are encountered while walking from $v_{j}$ to $v_{k}$. Finally, we construct a string $S^{+}=s_{1}^{+} \# s_{2}^{+} \# \ldots \# s_{n}^{+}$consisting of all the $s_{i}^{+}$'s separated by a symbol \#.

Lemma 7. Given an orthogonal representation $\Gamma$ with $b$ turns (bends) of a degree-4 biconnected graph $G$ with $n$ vertices, we can encode its turns using $(b+$ $m-1) \log _{2} 3 \approx 1.58(b+m-1)$ bits in $O\left((b+m)^{2}\right)$ time, and at most $1.67(b+m-1)$ 
bits in $O(b+m)$ time (using Huffman Encoding). These encodings can be decoded in $O\left((b+m)^{2}\right)$ and $O(b+m)$ time, respectively, to obtain the turns of $\Gamma$.

If $\Gamma$ is a turn-monotone orthogonal representation, then we can reduce the length of $S^{+}$by using Property 5 as follows: $c$ induces an ordering $f_{1}, f_{2}, \ldots, f_{p}$ of the internal faces of $G$, such that when we reconstruct $G$ using $c$, as in the proof of Theorem 2, starting from an initially empty graph, the faces will get inserted into it in the same order. Let $I_{1}, I_{2}, \ldots, I_{K}$ be the corresponding intervals of $c$. The ordering $f_{1}, f_{2}, \ldots, f_{p}$ is defined as follows (see Figure $1(\mathrm{~b})$ ): Let $I_{1}=\left[v_{3}, v_{3+q^{\prime}}\right]$, where $q^{\prime \prime} \geq 0$. Face $f_{1}$ is the face consisting of the vertices $v_{1}, v_{3}, \ldots, v_{3+q^{\prime}}, v_{2}$. In general, suppose we have already constructed the partial ordering $f_{1}, f_{2}, \ldots, f_{s}$ of the faces, using intervals $I_{1}, I_{2}, \ldots, I_{k-1}$. Let $I_{k}=\left[v_{k}, v_{k+q}\right]$, where $q \geq 0$. Let $P=v_{1}\left(=u_{1}\right) u_{2} \ldots u_{s}\left(=v_{2}\right)$ be the subpath of $H_{k-1}$ that we obtain by removing the edge $\left(v_{1}, v_{2}\right)$ from $H_{k-1}$. Let $C_{j}$ be the path $v_{k} v_{k+1} \ldots v_{k+q}$. We have 2 cases:

$-q>0$ : Then, $C_{j}$ has exactly two neighbors $v_{l}$ and $v_{r}$ in $H_{k-1}$. Let $x_{1}=$ $\left(v_{l}\right) x_{2}, \ldots, x_{t}\left(=v_{r}\right)$ be the subpath of $P$ that connects $v_{l}$ and $v_{r}$. Then, $f_{s+1}$ is the internal face of $G$ consisting of the vertices $v_{l}\left(=x_{1}\right), v_{k}, \ldots, v_{k+q}, v_{r}(=$ $\left.x_{t}\right), x_{t-1}, x_{t-2}, \ldots, x_{2}$. We say that face $f_{s+1}$ belongs to Interval $I_{k}$.

$-q=0$ : Then $C_{j}$ consists of exactly one vertex $v_{k}$. Let $u_{1}^{\prime}\left(=v_{l}\right), u_{2}^{\prime}, \ldots, u_{t}^{\prime}(=$

$\left.v_{r}\right)$ be the left-to-right order of the neighbors of $v_{k}$ in $H_{k-1}$. Let $P_{i}^{\prime}$, where $1 \leq i \leq t-1$, be the subpath of $P$ that connects $u_{i}^{\prime}$ and $u_{i+1}^{\prime}$. Then, each face $f_{s+i}$, where $1 \leq i \leq t-1$, is the internal face that consists of the vertex $v_{k}$ and the vertices of path $P_{i}^{\prime}$. We say that face $f_{s+i}$ belongs to Interval $I_{k}$.

(Figure 1(b) also shows the ordering of the faces given by the rightmost canonical ordering shown in Figure 1(b).) Let $T_{c}$ be the canonical spanning tree associated with $c$. For each face $f_{i}$, a tree edge of $f_{i}$ is one that is also an edge of $T_{c}$.

Fact 1. Except for one non-tree edge e, all the non-tree edges of each face $f_{i}$ are already contained in the faces $f_{1}, f_{2}, \ldots, f_{i-1}$. We will call edge $e$ as the non-tree completion edge of $f_{i}$.

Intuitively, we call the edge non-tree completion edge because, while reconstruct$\operatorname{ing} G$ using $c$, this is the only non-tree edge that we need to add to the already constructed graph to add face $f_{i}$ to it (of course, we will need to add the tree edges of $f_{i}$ also). For example, in Figure 1, edge $(14,12)$ is the non-tree completion edge of face $f_{10}$. For the face $f_{s}$, in the case $q>0$ given above, the non-tree completion edge is the edge $\left(v_{k+q}, v_{r}\right)$. For each face $f_{s+i}$, in the case $q=0$ given above, the non-tree completion edge is the edge $\left(v_{k}, u_{i+1}\right)$.

Since each edge of a turn-monotone orthogonal representation $\Gamma$ has same kinds of turns only (left or right, but not both), Property 5 implies that for any face of $\Gamma$, it is sufficient to encode the turns of all but one edge, namely its non-tree completion edge $e$, since the turns of $e$ can be deduced from the turns of the other edges. Infact, Lemma 8 says that it is sufficient to encode turns of tree edges: 
Lemma 8. Let $\Gamma$ be a turn-monotone orthogonal representation of a degree-4 biconnected plane graph $G$. let c be a rightmost canonical ordering of $G$. Suppose we construct a string $S^{+}$encoding the turns of $\Gamma$ as in Lemma 7 using c, except that $S^{+}$encodes the turns of only the tree edges of $G$. Then, by decoding $S^{+}$we can obtain the turns of all the edges of $\Gamma$.

Proof. Let $f_{1}, f_{2}, \ldots, f_{p}$ be the ordering of faces that corresponds to $c$, as defined above. We can easily prove this lemma can using induction:

Base Case: Consider face $f_{1}$. Decoding $S^{+}$will give us the turns of all the tree edges of $f_{1} \cdot f_{1}$ has exactly one non-tree edge $e$ (which is its non-tree completion edge). From Property 5, we can determine the turns of $e$ also.

Induction: Suppose we have already determined the turns of all the edges of faces $f_{1}, f_{2}, \ldots, f_{i-1}$. Consider face $f_{i}$. From Fact 1, except for its non-tree completion edge $e$, all the other non-tree of $f_{i}$ are already contained in the faces $f_{1}, f_{2}, \ldots, f_{i-1}$. Decoding $S^{+}$will give us the turns of all the tree edges of $f_{1}$. Hence, except for $e$, we would know the turns of all the edges of $f_{i}$. From Property [5], we can determine the turns of $e$ also.

Since, $T_{c}$ has exactly $n-1$ edges, we have:

Lemma 9. Given a turn-monotone orthogonal representation with b turns (bends) of a degree-4 biconnected graph with $n$ vertices, we can encode its turns using at most $(b+n-2) \log _{2} 3 \approx 1.58(b+n-2)$ bits in $O\left((b+n)^{2}\right)$ time, and at most $1.67(b+n-2)$ bits in $O(b+n)$ time (using Huffman Encoding). These encodings can be decoded in $O\left((b+n)^{2}\right)$ and $O(b+n)$ time, respectively, to obtain the turns.

To encode an orthogonal representation $\Gamma$, we construct a string $S_{1}=A^{\prime} L^{\prime} A^{*} L^{*} S^{\prime} S^{*} S^{+}$, where $S^{\prime}, S^{*}, S^{+}$are strings encoding structure, angles, and turns, respectively, of $\Gamma$, as given by Lemmas 1 (or 2] 3, or 44), 5 (or 6), and 7 (or 9), respectively, $L^{\prime}\left(L^{*}\right)$ is length of $S^{\prime}\left(S^{*}\right)$ in binary notation, and $A^{\prime}\left(A^{*}\right)$ encodes the length of $L^{\prime}\left(L^{*}\right)$ in unary notation, and consists of $\left|L^{\prime}\right|\left(\left|L^{*}\right|\right) 0^{\prime}$ 's followed by a 1 . Note that lengths of $A^{\prime}, L^{\prime}, A^{*}$, and $L^{*}$ are $O(\log n)$ each.

\section{$7 \quad$ Encoding Lengths of Line-Segments}

Let $d$ be a planar orthogonal drawing with $b$ turns of a degree- 4 biconnected plane graph $G$ with $n$ vertices and $m$ edges. Suppose each line-segment of $d$ has length at most $W$. Just as we constructed a string $S^{+}$in Section 6 to encode turns, we can construct a string $S^{\prime \prime}=s_{1}^{\prime \prime} s_{2}^{\prime \prime} \ldots s_{m}^{\prime \prime}$, where each $s_{i}$ contains the lengths of all the line-segments of edge $e_{i}=\left(v_{j}, v_{k}\right)$, placed in the order the corresponding linesegments are encountered while traveling along $e_{i}$ from $v_{j}$ to $v_{k}$, where $j<k$.

We can reduce the length of $S^{\prime \prime}$ by using the following property of $d$ : Suppose we orient each horizontal line-segment of $d$ as going "East" or "West", and each vertical line-segment as going "North" or "South", assuming that the linesegment of the edge $\left(v_{1}, v_{2}\right)$ incident on $v_{1}$ goes East. (We can easily do this in $O(n+m+b)=O(n+b)$ time using the angle and turn information contained in $d$.) 
Property 6. In $d$, for each face of $G$ :

1. The sum of the lengths of all the line-segments going East $=$ the sum of the lengths of all the line-segments going West, and

2. The sum of the lengths of all the line-segments going North $=$ the sum of the lengths of all the line-segments going South.

Property 6 implies that we can omit encoding the length of one horizontal and one vertical line-segment of $f_{i}$, and still be able to obtain the lengths of all the line-segments of $f_{i}$ from an encoding of the lengths of its other line-segments. To decide which line-segments to omit, consider the ordering $f_{1}, f_{2}, \ldots, f_{m}$ of the faces of $G$ that we can obtain from a rightmost ordering $c$ of $G$, as described in Section 6. Let $I_{k}=\left[v_{k}, v_{k+q}\right]$ be the interval of $c$, such that $f_{i}$ belongs to $I_{k}$. Let $E_{i}$ be the set of all the edges of $f_{i}$ that are not in the faces $f_{1}, f_{2}, \ldots, f_{i-1}$. Note that $E_{i}$ contains at least one edge, namely, the non-tree completion edge $e=$ $(u, v)$ of $f_{i}$. Moreover, the edges of $E_{i}$ form a connected path $p$, which connects $u$ with a vertex $u^{\prime}$, where $u$ is the end-vertex of $e$ that belongs to $H_{k-1}$, and $u^{\prime}$ is a vertex common to both $f_{i}$ and $f_{i-1}$. The free horizontal (vertical) line-segment of $f_{i}$ is defined as the first horizontal (vertical) line-segment encountered while traveling along $p$ from $u$ to $u^{\prime}$. Note that $f_{i}$ will have at least one free line-segment (which can be horizontal or vertical). While encoding the lengths of the linesegments of $d$, we can omit from $S^{\prime \prime}$ all the free line-segments of the faces of $G$.

Having constructed $S^{\prime \prime}$, we can construct a string $S_{2}=A^{\prime \prime} T^{\prime \prime} S^{\prime \prime}$, where $T^{\prime \prime}$ stores the value of $W$ in binary using exactly $\log _{2}(W+1)$ bits, and $A^{\prime \prime}$ contains a sequence of $\left|T^{\prime \prime}\right| 0$ 's followed by a 1 . String $A^{\prime \prime}$ basically encodes the length of $T^{\prime \prime}$ in unary notation. We have the following lemma:

Lemma 10. We can encode the lengths of the line-segments of d using a string $S_{2}$ consisting of $1+\left(2+b+m-f_{H}-f_{V}\right) \log _{2}(W+1) \leq 1+(2+b+m-f) \log _{2}(W+1)$ bits in $O\left(\left|S_{2}\right|\right)$ time, where $f_{H}$ and $f_{V}$ are the number of horizontal and vertical free line-segments, respectively, of $d$. Assuming that, while decoding, we already know all the angles and turns of $d$, we can decode $S_{2}$ to obtain the lengths of all the line-segments of $d$ in $O\left(\left|S_{2}\right|\right)$ time.

\section{Encoding a Planar Orthogonal Drawing}

Let $d$ be a planar orthogonal drawing of a degree-4 biconnected planar graph $G$. Let $\Gamma$ be the orthogonal representation of $G$ that corresponds to $d$.

We can encode $d$ by constructing a string $S=B L S_{1} S_{2}$, where $S_{1}$ is the string constructed in Section [6] that encodes $\Gamma, S_{2}$ is the string constructed using Lemma 10 that encodes the free lengths of the line-segments of $d, L$ is a string, with length $\log _{2}\left(\left|S_{1}\right|+1\right)$, that encodes in binary notation the length of string $S_{1}$, and $B$ is a string that contains a sequence of $|L| 0$ 's followed by a 1 . $B$ encodes the length of $L$ in unary notation.

We can obtain $d$ by decoding $S$, by first extracting $B$ from it and obtaining the length of $L$, then extracting $L$ and obtaining the length of $S_{1}$, then extracting 
$S_{1}$ and decoding it to obtain $\Gamma$, and finally, extracting $S_{2}$ and decoding it to obtain the lengths of the line-segments of $d$. This is summarized in Theorem 3

Theorem 3. Let $d$ be a planar orthogonal drawing, with $b$ turns (bends) of a plane graph $G$ with $n$ vertices, $m$ edges, and $f$ internal faces. Suppose each linesegment of $d$ has length at most $W$. Let $p=(2+b+m-f) \log _{2}(W+1)$. Let $q=n+b$.

- If $G$ is a degree-4 biconnected graph, then we can encode (and decode) d using at most $4.74 m+2.42 n+1.58 b+p+O(\log q)$ bits in $O\left((n+b+p)^{2}\right)$ time, and using at most $5.01 m+2.33 n+1.67 b+p+O(\log q)$ bits in $O(n+b+p)$ time.

- If $G$ is a degree-4 triconnected graph, then we can encode (and decode) d using at most $3.58 m+2.59 n+1.58 b+p+O(\log q)$ bits in $O\left((n+b+p)^{2}\right)$ time, and using at most $3.67 m+2.67 n+1.67 b+p+O(\log q)$ bits in $O(n+b+p)$ time.

- If $G$ is a degree-3 biconnected graph, then we can encode (and decode) d using at most $4.74 m+1.23 n+1.58 b+p+O(\log q)$ bits in $O\left((n+b+p)^{2}\right)$ time, and us ing at most $5.01 m+1.33 n+1.67 b+p+O(\log q)$ bits in $O(n+b+p)$ time.

- If $G$ is a degree-3 triconnected graph, then we can encode (and decode) d using at most $3.58 m+n+1.58 b+p+O(\log q)$ bits in $O\left((n+b+p)^{2}\right)$ time, and using at most $3.67 m+n+1.67 b+p+O(\log q)$ bits in $O(n+b+p)$ time.

Moreover, if $d$ is turn-monotone, then we can encode it using fewer bits, as follows:

- If $G$ is a degree-4 biconnected graph, then we can encode (and decode) d using at most $3.16 m+4 n+1.58 b+p+O(\log q)$ bits in $O\left((n+b+p)^{2}\right)$ time, and using at most $3.34 m+4 n+1.67 b+p+O(\log q)$ bits in $O(n+b+p)$ time.

- If $G$ is a degree-4 triconnected graph, then we can encode (and decode) d using at most $2 m+4.17 n+1.58 b+p+O(\log q)$ bits in $O\left((n+b+p)^{2}\right)$ time, and using at most $2 m+4.34 n+1.67 b+p+O(\log q)$ bits in $O(n+b+p)$ time.

- If $G$ is a degree-3 biconnected graph, then we can encode (and decode) d using at most $3.16 m+2.81 n+1.58 b+p+O(\log q)$ bits in $O\left((n+b+p)^{2}\right)$ time, and using at most $3.34 m+3 n+1.67 b+p+O(\log q)$ bits in $O(n+b+p)$ time.

- If $G$ is a degree-3 triconnected graph, then we can encode (and decode) d using at most $2 m+2.58 n+1.58 b+p+O(\log q)$ bits in $O\left((n+b+p)^{2}\right)$ time, and using at most $2 m+2.67 n+1.67 b+p+O(\log q)$ bits in $O(n+b+p)$ time.

\section{References}

1. R. C. Chuang, A. Garg, X. He, M. Y. Kao, and H. Lu. Compact encoding of planar graphs via canonical ordering and multiple parenthesis. In Proc. International Colloqium on Automata, Languages and Programming (ICALP), pp. 118-129, 1998.

2. H. de Fraysseix, J. Pach, and R. Pollack. How to draw a planar graph on a grid. Combinatorica, 10(1):41-51, 1990.

3. G. Di Battista, G. Liotta, and F. Vargiu. Diagram Server. J. Visual Lang. Comput., 6(3):275-298, 1995 
4. G. Di Battista, G. Liotta, and F. Vargiu. Spirality and optimal orthogonal drawings. SIAM J. Comput., 27(6):1764-1811, 1998.

5. Xin He, M.-Y. Kao, and H. Lu. A fast general methodology for informationtheoretically optimal encodings of graphs. SIAM J. Comput., 30(3):838-846, 2000.

6. G. Kant. Drawing planar graphs using the lmc-ordering. In Proc. 33th Annu. IEEE Sympos. Found. Comput. Sci., pages 101-110, 1992.

7. R. Tamassia. On embedding a graph in the grid with the minimum number of bends. SIAM J. Comput., 16(3):421-444, 1987.

8. R. Tamassia and I. G. Tollis. Planar grid embedding in linear time. IEEE Trans. Circuits Syst., CAS-36(9):1230-1234, 1989.

9. W. T. Tutte. A census of planar triangulation. Canad. J. Math., 14:21-38, 1962. 